\title{
IMPROVEMENT OF MPLS PERFORMANCE BY IMPLEMENTATION OF A MULTI- AGENT SYSTEM
}

Rana Rahim-Amoud, Leila Merghem-Boulahia, and Dominique Gaiti

ISTIT, University of Technology of Troyes, 12 rue Marie Curie, BP 2060, 10000 Troyes Cedex, France

Abstract: $\quad$ Multi-Protocol Label Switching (MPLS) is a network layer packet forwarding technology that provides flexible circuit switched traffic engineering solutions in packet switched networks by explicit path routing. However, the actual weakness of MPLS resides in its inability to provide application-level routing intelligence, which is a fundamental component especially for voice delivery. In this paper we propose to introduce a Multi-Agent System (MAS) within the MPLS network to improve its performance. The introduction of agents takes place into the decision points in MPLS at the flow level, and distributes traffic based on the quality of service required by the type of traffic. We also propose an intelligent framework for network as well as an architecture of our agent in order to improve the efficiency of the Quality of Service (QoS) within MPLS.

Keywords: MPLS; Quality of Service (QoS); Multi-Agent Systems (MAS); Artificial Intelligence (AI).

\section{INTRODUCTION}

The usage of the Internet has increased enormously in the last few years. At the same time, new real-time as well as non-real-time applications have emerged demanding for much improved Quality of Service (QoS) than the best effort currently proposed by the Internet. To adapt the network functioning to the requirements of these different types of applications, different networking solutions were proposed, such as the Integrated Services (IntServ) ${ }^{1}$, its Resources reSerVation Protocol (RSVP) ${ }^{2}$, and the Differentiated Services (DiffServ) ${ }^{3}$. Other proposals, like Active Networks 
$(\mathrm{AN})^{4}$, introducing an adaptive management plane which allows real-time configuration management ${ }^{5}$ and Multi-Protocol Label Switching, each within its objectives, have all succeeded in introducing amelioration to the global functioning of the network. They, however, still face miscellaneous limitations. To overcome these limitations, it became essential to find a single solution which makes it possible to exploit, to the maximum, the advantages of the existing solutions while playing the role of an essential complement. The evolution of the networks is marked by a tendency towards the intelligence and autonomy, removing any kind of centralization of the decisions, and opening the doors towards self-management and selfchecking while ensuring the scalability, the adaptability and the survival of the networks. In line with this, recent research showed the effectiveness, the reliability and the robustness of the Multi-Agent System (MAS) for the dynamic management of complex and distributed systems. Our goal is to find the decision points in which we could introduce our intelligent agents, allowing as a result an improvement of the network performance and a satisfaction of users' requests. We chose for our study the Multi-Protocol Label Switching (MPLS) network which provides a set of services (Virtual Private Networks VPN and Traffic Engineering TE) in addition to the fast commutation that it allows between routers.

The paper is organized as follows. We first introduce MPLS protocol, its weakness and the current researches around this area. We then propose to find the decision points. In section 4 , we propose an intelligent framework as well as an architecture of our agent. Finally we make a brief conclusion.

\section{MPLS PROTOCOL}

MPLS ${ }^{6}$ is a new technology that associates labels with routers and uses these labels to forward packets by specifying the Forwarding Equivalence Class (FEC). FEC is a representation of a group of packets that share the same requirements for their transport. All packets in such a group receive the same treatment in the domain. MPLS domain contains two types of equipments LER (Label Edge Router) and LSR (Label Switch Router). The LERs are also called I-LSR (Ingress LSR) for the LSR that puts the label to an incoming packet and E-LSR (Egress LSR) to the one which removes the label from the outgoing packet to return it to its initial nature. LSR is a highspeed router device in the core of the MPLS network. In practice, the labels are distributed from the initialization of the network, related to the network configuration and routing tables which are established by a classic protocol like IGP. The path between 2 LERs is called LSP (Label Switched Path). As opposed to conventional IP forwarding, in MPLS, the assignment of a 
System

particular packet to a particular FEC is done just once, as the packet enters the network ${ }^{7}$. Many protocols can be used to distribute labels, LDP (Label Distribution Protocol) $)^{8}$, CR-LDP (Constraint based-LDP) ${ }^{9}$ that is an extension of LDP and RSVP-TE (RSVP with Traffic Engineering) ${ }^{10}$. LDP is a peer-to-peer protocol, while CR-LDP and RSVP-TE provide mechanisms for establishing end-to-end explicitly routed LSPs.

\subsection{The weakness of MPLS}

Currently the weakness of MPLS resides in its inability to provide application-level routing intelligence, which is a fundamental component especially for voice delivery ${ }^{11}$. Voice over IP (VoIP) is a critical application that requires intelligent routing alternation on the call level to prevent latency, delay, packet loss and jitter and this cannot be provided by MPLS. Adapting MPLS to VoIP traffic necessitates the distinction of different traffic types. Some solutions, which couple MPLS with DiffServ or RSVP, were proposed to solve MPLS limitations. According to ${ }^{11}$, DiffServ over MPLS solution "does nothing to solve the static route problem of MPLS". If the QoS on a route degrades, MPLS plus DiffServ will not change the route, while it introduces complexity into the architecture. The implementation of RSVP with MPLS prevents an overbooking in the router from the start. However, RSVP "is ineffective and impractical for solving the fluctuating demands of VoIP" ${ }^{\prime \prime}$. Even with these proposed solutions, MPLS remains unable to guarantee the QoS of incoming traffic, so it is very essential to find another solution.

\section{$2.2 \quad$ Current researches}

One of the critical research issues in MPLS is an efficient mapping of the traffic flows to LSPs. Such mapping provides an effective and efficient use of network resources and enables End-to-End QoS routing for real-time traffic. Having multiple LSPs for a destination is a typical setting which exists in an operational Internet Service Provider (ISP) network that implements MPLS technology. With multiple LSPs available for an egress node, the goal of the ingress node is to distribute the traffic across the LSPs by selecting the appropriate LSP from the available ones. Consequently, the network utilization as well as the network performance perceived by users are enhanced. According to this, Song et al. ${ }^{12}$ discuss Load Distribution Management (LDM). Their main goal is to enhance the network utilization as well as the network performance by adaptively splitting traffic load among multiple paths. An LSP for an incoming traffic flow is dynamically selected based on both the current congestion level, and the length of the 
path in terms of the number of hops. LDM is intended for the best-effort traffic that does not impose any particular service requirement to the network. While LDM provides load-balancing solution and an efficient congestion control mechanism with increased utilization, it does not look into service differentiation for time-sensitive traffics like voice and video. Patek et al. ${ }^{13}$ propose a simple alternate routing (SAR) in order to make a dynamic routing for aggregate traffic. SAR is based on three different parts: congestion discovery, selection of alternate paths, and allocation of traffic along alternate paths. Border nodes are responsible for satisfying all these three parts. The goal in SAR is to reroute traffic around congestion. SAR supposes that the differentiated services of the incoming traffic are already done and treats only the second phase. These above solutions remain unable to satisfy and to guarantee the QoS required for incoming traffic. We propose to introduce a MAS within the MPLS network to improve its performance and to guarantee a differentiation of services of incoming traffic. So we begin by finding the decision points.

\section{DECISION POINTS}

The first step of our research is to find the decision points. Once found, we can add our intelligent agents within these points.

\subsection{The first decision point}

Let us examine what occurs in the entry of the MPLS domain. Different types of traffic flows arrive to the entry of the MPLS domain. The classification of the packets is done just at the entry of the domain by I-LSR, by assigning a particular packet to a particular FEC. Within the domain, there is no reclassification, packets are just switched. Currently the most usable criteria to build FECs is based on the destination address or the prefix of the destination address, by taking advantage from the aggregation of flows that have the same destination. Aggregation may reduce the number of labels which are needed to handle a particular set of packets. It may also reduce the amount of needed label distribution control traffic. However, it does not take into account the type of traffic. Echchelh ${ }^{14}$ has demonstrated by simulations with QNAP2 that the aggregation of different characteristics flows within the MPLS domain degrades the performances. Aggregation must be, consequently, based on the type of traffic and the required quality of service. In this paper we propose to introduce an intelligent agent on the level of each I-LSR router which is an efficient and a pertinent decision point. This agent will have as a role to examine the incoming flows and to create for each type of traffic a different FEC and consequently a different 
System

LSP, even for traffics which have the same destination. As each E-LSR is at the same time an I-LSR for the packets forwarded in the opposite direction, then our intelligent agent will be introduced on the level of each LER within the MPLS domain, and consequently we obtain a MAS (Fig. 1). MPLS, in conjunction with path establishment protocols such as CR-LDP or RSVPTE, makes it possible to set up a number of LSPs between a sourcedestination pair $^{6,9,15}$. MPLS, with the efficient support of explicit routings enables assigning a particular traffic stream onto one of the available LSPs providing basic mechanism for facilitating traffic engineering.

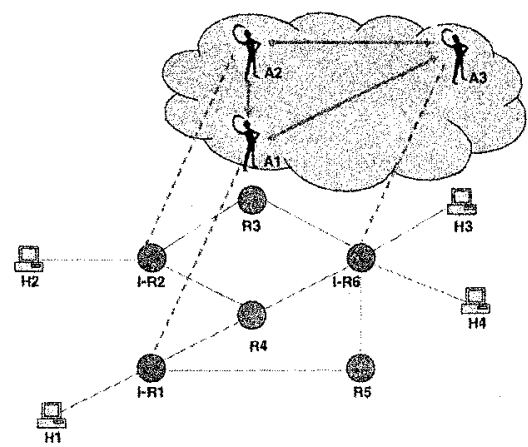

Figure 1. An intelligent agent is introduced on the level of each LER

The purpose of the introduction of our intelligent agent is to examine the current state of the network as well as incoming flows to, firstly select the suitable algorithm to start at the suitable moment and secondly to change the parameters of the selected algorithm.

\subsection{The second decision point}

According to ${ }^{6}$, an LSR is capable of label merging if it can receive two packets from different incoming interfaces, and/or with different labels, and send both packets on the same outgoing interface with the same label. The MPLS architecture accommodates both merging and non-merging LSRs. In addition, MPLS contains procedures to ensure correct interoperation between them. To illustrate the importance of label merging, let us consider the example shown in Fig. 2. In the event that an LSR in the middle of the MPLS domain (R6) is merge-capable, the LSR simply acts as an Egress LSR to upstream neighbors and as Merge LSR to downstream neighbors ${ }^{16}$.In the example (Fig. 2), if LSR R6 were merge-capable, it would perform aggregation of requests from upstream neighbors $\mathrm{R} 1$ and $\mathrm{R} 2$ thus reducing label consumption within the MPLS Network, the only condition is that the 
flows must be in the same FEC. Furthermore, label merging causes a small delay because it is designed for delay-insensitive traffic. Our proposal is to introduce an intelligent agent on the level of the LSR R6 having for role to activate the merge-capable of R6 when traffic is not delay-sensitive and to deactivate it in other cases. As a consequence the two LSPs will be aggregated into only one LSP from the point of intersection of the two LSPs.

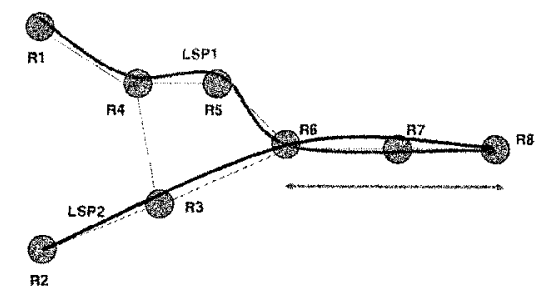

Figure 2. LSP attribution before the introduction of the intelligent agent.

\section{AGENT AND MULTI-AGENT SYSTEMS}

\subsection{Definition}

Agents and MAS are two innovative and interesting concepts for a great number of researchers in different domains such as simulation of road traffic $^{17-19}$, simulation of social phenomena ${ }^{20,21}$, simulation of biological phenomena ${ }^{22-24}$, medical simulation ${ }^{25}$, etc. According to ${ }^{26}$, an agent is a physical or virtual entity having trends and resources, able to perceive its environment, to act on it and to acquire a partial representation of it. It is also able to communicate with other peers and devices, and has a behavior that fits its objectives according to its knowledge and capabilities. The most important thing is that agents can learn, plan future tasks and are able to react and to change their behavior according to the changes in their environment. A MAS is a group of agents able to interact and cooperate in order to reach a specific objective. Agents are characterized by their properties that determine their capabilities. Different properties are defined like autonomy, proactive-ness, flexibility, adaptability, ability to collaborate and coordinate tasks and mobility. According to its role within its environment, the agent acquires some of these properties.

\subsection{Agents and Multi-Agent System Architecture}

Agents can be reactive, cognitive, hybrid or adaptive $e^{24,27,28}$. Reactive agents are suitable for situations where we need less treatment and faster responses 

System

(actions). Cognitive agents, on the other side, allow making decisions and planning based on deliberations taking into account the knowledge of the agent about itself and the others. Adaptive agents can adapt their actions and parameters to the changing situations. Hybrid agents are composed of several concurrent layers. Our approach is based on the architecture developed $\mathrm{in}^{29}$. In this architecture, two levels of agents are defined: the first one is composed of one agent called Master Agent which is a cognitive agent while the second one is composed of several reactive agents: LDP agent, Aggregation Agent, Queue Manager Agent, Routing Agent, Admission Controller Agent, etc (Fig. 3).

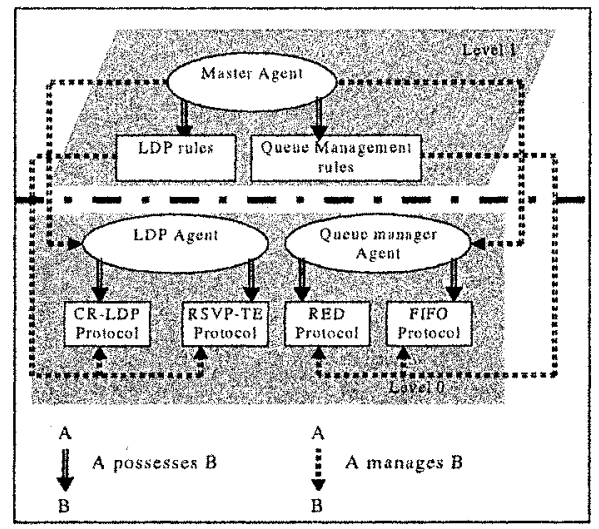

Figure 3. Two levels MAS architecture

The Master Agent observes the current router conditions and chooses the most appropriate protocols to the other agents under its responsibility. Each agent of level 0 has a set of protocols dedicated to the task it is in charge of. For example, LDP agent establishes some rules for selecting which label distribution protocol (LDP, CR-LDP, RSVP-TE...) to use under which circumstances. Deciding on what protocol is the most appropriate (and which must be adopted) will depend on the current QoS measures (loss, delay and jitter). In fact, there is no protocol giving optimal performance whatever the network condition. Hence, the need to adapt the current protocols to this new situation. Each Master Agent possesses a set of rules allowing to select the appropriate protocols to activate, and therefore to select the best actions to execute. These rules give the node the means to guarantee that the set of actions executed, at every moment, by its agents are coherent, in addition to be the most relevant to the current situation ${ }^{30}$. In order to minimize conflict situations, rules are organized in separate modules following the task they are interested (Fig. 3 shows an example). 


\subsection{Actions of the Master Agent}

The actions of the Master Agent may consist in:

- letting the protocol running: this occurs when the protocol is still relevant to the current conditions;

- modifying the internal functioning of the protocol: this modification appears by updating the parameters on which the protocol depends;

- inhibiting the protocol: the inhibition happens when this protocol becomes useless regarding the current node's situation and rules;

- activating the protocol: the activation takes place if the Master Agent considers that this protocol is appropriate to the current node conditions. This activation may be accompanied by the inhibition of other protocols to avoid the coexistence of contradictory protocols.

The actions undertaken by the node have local consequences but may influence the decisions of the other nodes. In fact, by sending messages bringing new information on the sender node's state, a receiver's Master Agent rule may be triggered. This can involve a change within the receiver node (the inhibition or the activation of a protocol, etc.). This change may have repercussions on other nodes, and so forth until the entire network to be affected. This dynamic process aims to adapt the network to new conditions and takes advantage of the agents' abilities to alleviate the global system. We argue that these agents will achieve an optimal adaptive management process because of the following two points: (1) each agent holds different processes (protocols and adaptive selection of these protocols) allowing to take the most relevant decision at every moment; (2) the agents are implicitly cooperative in the sense that they possess rules that take account of the neighbors' state in the process of protocols' selection.

\section{CONCLUSION}

In this paper, we proposed to introduce a MAS within the MPLS domain in order to improve its performance. One of the step consisted of finding the decision points into MPLS which are especially identified on the entry of the domain on the I-LSR routers. The MAS is then situated into these decision points. The MAS has as role to set up multiple LSPs between an ingressegress pair, and to distribute dynamically the incoming traffics to these LSPs. Traffics are distributed basing on their type and the required QoS. A basically two-layers architecture of MAS is also proposed in this paper. This architecture aims to dynamically select the appropriate protocol following the current QoS parameters. As future work, we intend to improve our architecture, to define our adaptation rules and to make a testbed. 


\section{References}

1. R. Braden, D. Clark, and S. Shenker, Integrated Services in the Internet Architecture: an Overview, RFC1633 (1994).

2. J. Wroclawski, The Use of RSVP with IETF Integrated Services, RFC2210 (1997).

3. S. Blake, D. Black, M. Carlson, E. Davies, Z. Wang, and W. Weiss, Architecture for Differentiated Services, RFC2475 (1998).

4. D. Tennenhouse, J. Smith, D. Sincoskie, D. Wetherall, and G. Minden, A Survey of Active Network Research, IEEE Communications Magazine 35(1), 80-86 (1997).

5. M. De Castro, Programmable and Adaptive Management of $Q O S$ in IP networks, Ph.D thesis, (INT, France 2004).

6. E. Rosen, A. Viswanathan, and R. Callon, Multiprotocol Label Switching Architecture, RFC3031 (2001).

7. The international Engineering Consortium; http://www.iec.org.

8. L. Anderson, P. Doolan, N. Feldman, A. Fredette, and B. Thomas, LDP Specification, Network Working Group, RFC3036 (2001); http://www.ietf.org/rfc/rfc3036.txt.

9. J. Ash, M. Girish, E. Gray, B. Jamoussi, and G. Wright, Applicability Statement for CR-LDP, Network Working Group, RFC3036 (2002); http://www.ietf.org/rfc/rfc3036.txt.

10. D. Awduche, L. Berger, D. Gan, T. Li, V. Srinivaan, and G. Swallow, RSVP-TE Extensions to RSVP for LSP Tunnels, Network Working Group, RFC3209 (2001); http://www.ietf.org/rfe/rfc3209.txt.

11. PRIMEDIA Business Magazines \& Media Inc. (2003); http://infocus.telephonyonline.com/ar/telecom_questioning_mpls/.

12. J. H. Song, S. Kim, and M. J. Lee, Dynamic load distribution in MPLS networks, Information Networking 2662, 989-999 (2003).

13. S. D. Patek, R. Venkateswaran, and J. Liebeherr, Simple alternate routing for differentiated services networks, Computer Networks: the International Journal of Computer and Telecommunications Networking 37, 447-466 (2001).

14. Z. Echchelh, QoS and Allocation of resources in ATM and MPLS networks, Ph.D thesis (in French). (University of Bourgogne, France, 2001). 
15. B. Jamoussi, L. Andersson, R. Dantu, L. Wu, P. Doolan, T. Worster, N. Feldman, A. Fredette, M. Girish, E. Gray, J. Heinanen, T. Kilty, and A. Malis, ConstraintBased LSP set up using LDP, RFC 3212 (2002).

16. A. Fredette, C. White, L. Andersson, and P. Doolan, Stream Aggregation, Work in progress (1997); draft-fredette-mpls-aggregation-00.txt.

17. S. El Hadouaj, A. Drogoul, and S. Espié, How to Combine Reactivity and Anticipation: the Case of Conflicts Resolution in Simulated Road Traffic, MABS'2000 workshop 1979, 82-96 (Boston, USA, 2000).

18. A. Moukas, K. Chandrinos, and P. Maes: Trafficopter, A Distributed Collection System for Traffic Information, ClA'98 1435, 34-43 (Paris, France, 1998).

19. A.L.C. Bazzan, J. Wahle, and F. Klügl, Agents in Traffic Modelling - From Reactive to Social Behaviour, KI'99 1701, 303-307 (Bonn, Germany, 1999).

20. L. Ben Said, T. Bouron, and A. Drogoul, Agent-based interaction analysis of consumer behavior, AAMAS' 2002, ACM, 184-190 (Bologna, Italy, 2002).

21. R. Conte, N. Gilbert, and J.S. Sichman, MAS and Social Simulation: A Suitable Commitment, MABS'98 1534, 1-9 (Paris, France, 1998).

22. A. Drogoul, When ants play chess, From reaction to cognition 957 , edited by $\mathrm{C}$. Castelfranchi and J.P. Müller (Springer-Verlag, Berlin-Heidelberg, 1995), pp. 1327.

23. A. Pave, F. Bousquet, C. Cambier, C. Mullon, P. Morand, and J. Quensiere, Simulating the Interaction between a Society and a Renewable Resource, Journal of Biological Systems 1, 199-213 (1993).

24. J. Doran, Agent-Based Modelling of EcoSystems for Sustainable Resource Management, $3^{\text {rd }}$ EASSS'01 2086, 383-403 (Prague, Czech Republic, 2001).

25. P. Ballet, F. Harrouet, and J. Tisseau, A Multi-Agent System to model a Human Secondary Immune Response. IEEE International Conference on Systems, Man and Cybernetics (SMC'97), 357-362 (Orlando, USA, 1997).

26. J. Ferber, Multi-Agent System: An Introduction to Distributed Artificial Intelligence (Harlow: Addison Wesley Longman, 1999).

27. S. Barber, and C. Martin, Dynamic Adaptive Autonomy in Multiagent Systems, Representation and Justification, IJPR\&AI 15(3), 405-433 (2001).

28. M. Wooldridge, Intelligent Agents, in: Multiagent Systems: a Modern Approach to Distributed Artificial Intelligence, (Weiss G. Press, 1999), pp. 27-77.

29. L. Merghem, and H. Lecarpentier, Agents: A Solution for Telecommunication Network Simulation, Network Control and Engineering for QoS, Security and Mobility (NetCon'2002), 165-176 (Kluwer Academic publishers, Paris, France, 2002).

30. L. Merghem, D. Gaiiti, and G. Pujolle, On Using Agents in End to End Adaptive Monitoring, E2EMon Workshop, in conjunction with MMNS'2003 2839, 422-435 (Belfast, Northern Ireland, 2003). 